\title{
Efficacy and tolerance of lactitol supplementation for adult constipation: a systematic review and meta-analysis
}

This article was published in the following Dove Press journal:

Clinical and Experimental Gastroenterology

12 July 2014

Number of times this article has been viewed

Larry E Miller'

Julia Tennilä ${ }^{2}$

Arthur C Ouwehand ${ }^{2}$

'Miller Scientific Consulting, Inc., Asheville, North Carolina, USA;

${ }^{2}$ Danisco Sweeteners Oy, Active Nutrition, Kantvik, Finland
Correspondence: Larry E Miller Miller Scientific Consulting, Inc., I854 Hendersonville Road,

\#23I, Asheville, NC 28803, USA

$\mathrm{Tel}+\mathrm{I} 8284501895$

Fax +I 9282683563

Email larry@millerscientific.com
Background: Constipation is a common complaint in adults. Lactitol is an osmotic disaccharide laxative that increases fecal volume and stimulates peristalsis. In this paper, we present the first meta-analysis on the efficacy and tolerance of lactitol for adult constipation.

Methods: We searched MEDLINE ${ }^{\circledR}$ and Embase, with no date or language restrictions, for studies of lactitol supplementation on adult constipation. A random-effects meta-analysis was performed on pre- to posttreatment changes in stool frequency and consistency with lactitol among all studies, as well as a comparison of efficacy and tolerance outcomes in randomized controlled trials (RCTs) of lactitol versus lactulose.

Results: A total of eleven studies representing 663 distinct patients were included in the final analysis, including five single-arm studies, four RCTs comparing lactitol with lactulose, one RCT comparing lactitol with placebo, and one nonrandomized controlled trial comparing lactitol with stimulant laxatives. Weekly stool frequency was significantly increased with lactitol compared with baseline (standardized mean difference [SMD]: 1.56, $P<0.001$ ). Stool consistency also improved over the supplementation period with lactitol (SMD: 1.04, $P<0.001$ ). Approximately one-third of patients experienced an adverse event; however, symptoms were generally mild and rarely (5\%) resulted in study withdrawal. In RCTs of lactitol versus lactulose, lactitol was slightly more effective than lactulose in increasing weekly stool frequency (SMD: 0.19, $P=0.06$ ). No statistically significant differences between lactitol and lactulose were identified in any other efficacy or tolerance outcome. Lactitol demonstrated favorable efficacy and tolerance in individual studies when compared to stimulant laxatives and placebo.

Conclusion: Lactitol supplementation is well tolerated and improves symptoms of adult constipation. The efficacy and tolerance of lactitol and lactulose are similar, with a trend for more frequent stools with lactitol. Limited evidence suggests lactitol is superior to stimulant laxatives and placebo for relieving constipation symptoms.

Keywords: adult, bowel function, constipation, gastrointestinal, lactitol, laxative, osmotic, stool, sugar alcohol

\section{Introduction}

Constipation affects $2 \%$ to $27 \%$ of the adult population ${ }^{1,2}$ and disproportionately affects females and the elderly. ${ }^{3}$ Constipation symptoms are quite diverse but typically include infrequent bowel movements, hard or lumpy stools, excessive straining, bloating, abdominal discomfort, and/or feelings of incomplete evacuation. ${ }^{4}$ Adult constipation negatively influences health-related quality of life $\mathrm{e}^{5}$ and is responsible for a substantial patient and societal economic burden. ${ }^{6}$ The pathophysiology of constipation is poorly understood and likely multifactorial. Primary causes of constipation include intrinsic colonic or anorectal dysfunction, with secondary causes related to organic disease, 
systemic disease, or medications. ${ }^{7}$ Consequently, a wide variety of prescription and nonprescription medications is available for constipation, encompassing a diverse range of therapeutic targets and mechanisms of action. Nevertheless, half of patients remain unsatisfied with available treatment options, ${ }^{8}$ thereby highlighting the continued therapeutic gap in this patient population.

Osmotic laxatives are often prescribed as a first-line therapy in the management of constipation in children and adults. 9,10 "Lactitol", produced by hydrogenation of lactose, is an osmotic disaccharide laxative that increases osmotic pressure in the intestinal lumen, resulting in increased fecal volume and stimulation of peristalsis. ${ }^{11}$ Several clinical trials have been conducted on the efficacy and tolerance of lactitol in the treatment of adult constipation. However, no concerted effort has been made to synthesize and quantify these outcomes. The objective of this systematic review and meta-analysis was to determine the efficacy and tolerance of lactitol in the treatment of adult constipation.

\section{Methods}

\section{Search strategy}

We searched MEDLINE ${ }^{\circledR}$ and Embase, with no date or language restrictions, for studies of lactitol supplementation on adult constipation, regardless of study design. We used the search terms "Importal", "lactitol", "osmotic laxative", or "sugar alcohol" combined with "bowel", "constipat*", "gastrointestinal", "stool", or "transit" to identify relevant studies. References of included papers and relevant review articles were manually searched.

\section{Study selection}

Studies were included if they were published in a peerreviewed journal, investigated the influence of lactitol supplementation on constipation symptoms, included patients 18 years or older, and reported at least one efficacy or tolerance outcome. Studies were excluded if constipation was attributed to surgery, hospitalization, or organic gastrointestinal disease.

\section{Data extraction}

One investigator (LM) initially assessed study eligibility. Data were extracted and entered into a prospectively developed database and the entries were confirmed by a second investigator (AO). Disagreements were settled by discussion. The last search was performed in November 2013.
The following data were extracted: general manuscript information, study design characteristics (study design, comparison, sample size, constipation definition, lactitol delivery method and dosing schedule, treatment duration, concomitant therapies), patient characteristics (age, sex, body mass index, symptom duration), efficacy outcomes (stool frequency, stool consistency, intestinal transit time, physician- and patient-reported efficacy), and tolerance outcomes, including adverse events (AEs), AE-related withdrawals, withdrawals for any reason, and serious adverse events (SAEs).

\section{Statistical analysis}

A random-effects meta-analysis model was selected a priori given differences in study design characteristics, lactitol dosage and duration, and patient characteristics among studies. The standardized mean difference (SMD) and 95\% confidence interval (CI) was preferentially used to report continuous and interval outcomes due to differences in reporting scales among studies. SMD values of 0.2, 0.5, 0.8, and 1.0 represent small, medium, large, and very large effect sizes, respectively. ${ }^{12}$ We additionally reported the difference in means for weekly stool frequency only, since this outcome was reported in a consistent fashion in most studies. Frequencies and odds ratios with 95\% CIs were used to report binary outcomes. The $I^{2}$ statistic was used to estimate heterogeneity of treatment effects across studies with values of $\leq 25 \%, 50 \%$, and $\geq 75 \%$, representing low, moderate, and high inconsistency, respectively. ${ }^{13}$ Publication bias was assessed using Egger's regression test. ${ }^{14}$ Univariate meta-regression and subgroup analysis were performed to explore the influence of individual moderators on the pre- to posttreatment SMD of weekly stool frequency with lactitol. These analyses were not performed for other outcomes or for controlled studies due to the insufficient number of reported treatment effects. All analyses were performed using Comprehensive Meta-Analysis software (version 2.2; Biostat, Englewood, NJ, USA).

\section{Results Study selection}

Of 88 potentially relevant articles, 62 were considered irrelevant due to non-constipated patients, no lactitol supplementation group, or article type of review, case report, or correspondence. The full text of 26 papers was retrieved for further review. We excluded 15 studies for reasons shown in Figure 1. Ultimately, eleven studies, ${ }^{15-25}$ representing 663 distinct patients, were included in the meta-analysis. 


\section{Patient characteristics}

All studies enrolled adult patients with constipation not attributable to surgery, hospitalization, or organic gastrointestinal disease. Nine studies ${ }^{15-17,19-24}$ enrolled patients with chronic constipation (minimum duration $>6$ months). The minimum constipation symptom duration was 3 weeks in the study of Goovaerts and Ravelli ${ }^{18}$ and 3 days in the study of Xu et al. ${ }^{25}$ Mean age ranged from 37 to 84 years, mean body mass index ranged from 20 to $25 \mathrm{~kg} / \mathrm{m}^{2}$ (reported in five studies), and $72 \%$ of patients were female (Table 1 ).

\section{Study characteristics}

Of the eleven included studies, five were single-arm studies, ${ }^{15,16,18,21,24}$ four were randomized controlled trials (RCTs) comparing lactitol with lactulose, ${ }^{17,19,20,25}$ one was an RCT comparing lactitol with placebo, ${ }^{22}$ and one was a nonrandomized controlled trial comparing lactitol with stimulant laxatives. ${ }^{23}$ Total sample sizes ranged from 10 to 129 patients among studies. Lactitol was administered in powder form in all studies at a median starting dose of 20 g per day (range: 10-40 g per day) over a median treatment period of 28 days (range: 7-156 days). All studies utilized an individualized dosing regimen, which was adjusted over the treatment period according to patient response. Median dosing across the treatment period was $20 \mathrm{~g}$ per day (range: $11-35 \mathrm{~g}$ per day). No concomitant therapies were administered in any study, with the exception of laxatives for rescue therapy as needed.

\section{All studies: pre- to posttreatment lactitol effects}

Lactitol ingestion significantly increased weekly stool frequency compared with baseline (SMD: $1.56,95 \% \mathrm{CI}$ : 1.00-2.11, $P<0.001$; Figure 2), with a difference in means of 3.8 (95\% CI: $3.2-4.3, P<0.001)$ stools per week. Stool consistency also improved over the supplementation period with lactitol (SMD: 1.04, 95\% CI: 0.49-1.59, $P<0.001$; Figure 3). There was significant heterogeneity for stool frequency $\left(I^{2}=91 \%\right)$ and consistency $\left(I^{2}=87 \%\right)$ outcomes with lactitol among studies, with publication bias evident for stool consistency only. No included studies reported changes in intestinal transit time. Four studies reported subjective efficacy assessments. ${ }^{15,18,19,24}$ Overall, 77\% (209/272) of physician assessments and $72 \%(113 / 156)$ of patients rated lactitol efficacy as good or excellent.

Compliance with the lactitol supplementation regimen was excellent, with $93 \%(500 / 539)$ of patients completing the study. Approximately one-third of patients (173/509) experienced an AE; however, symptoms were generally mild with AEs and rarely $(5 \% ; 28 / 539)$ resulted in study withdrawal. Two SAEs (myocardial infarction and pulmonary embolism) were reported in a study of 14 elderly (mean age: 82 years) patients with multiple comorbidities residing in a long-term care facility. ${ }^{16}$ The relationship of lactitol use to the SAEs was not reported. No other study reported an $\mathrm{SAE}$ in any patient.

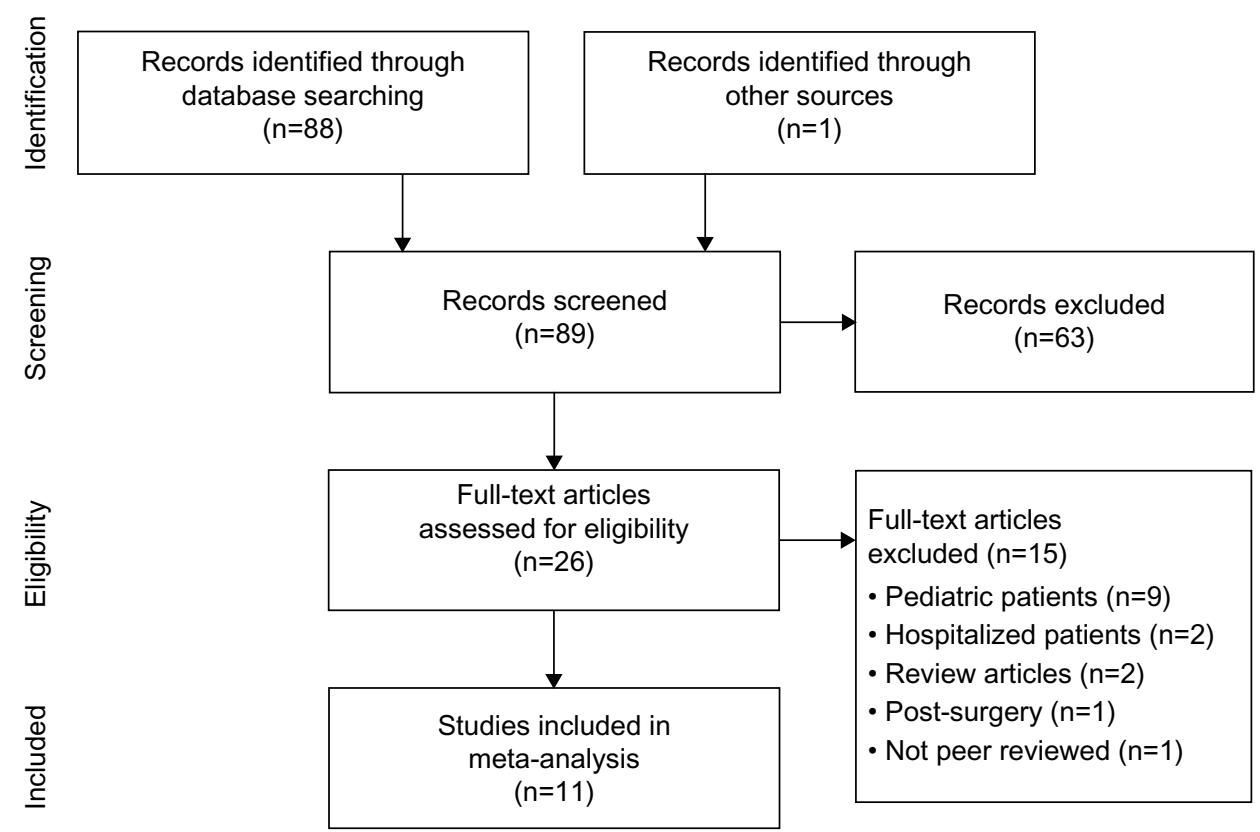

Figure I Preferred Reporting Items for Systematic Reviews and Meta-Analyses (PRISMA) flow diagram. 


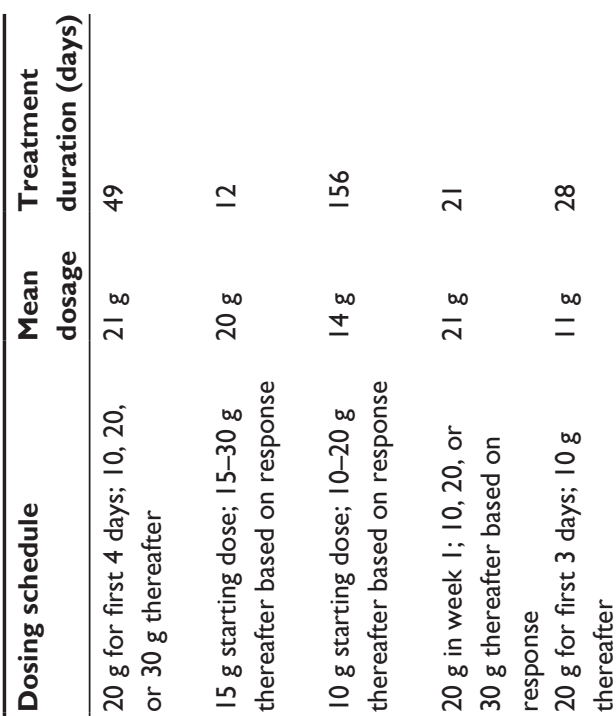

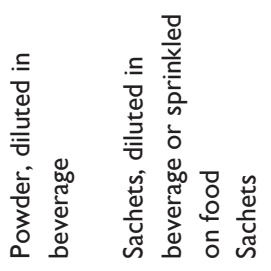

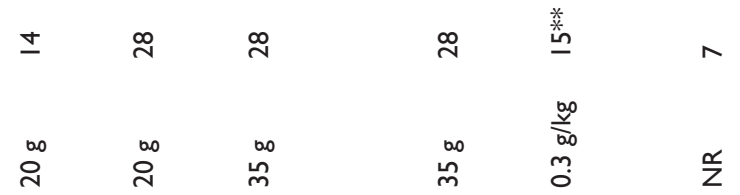

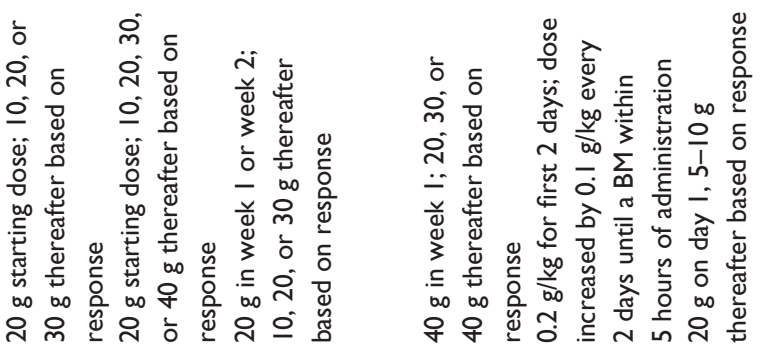

०.
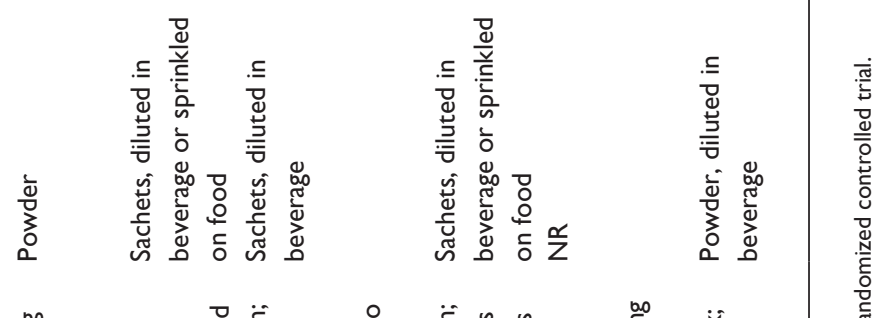

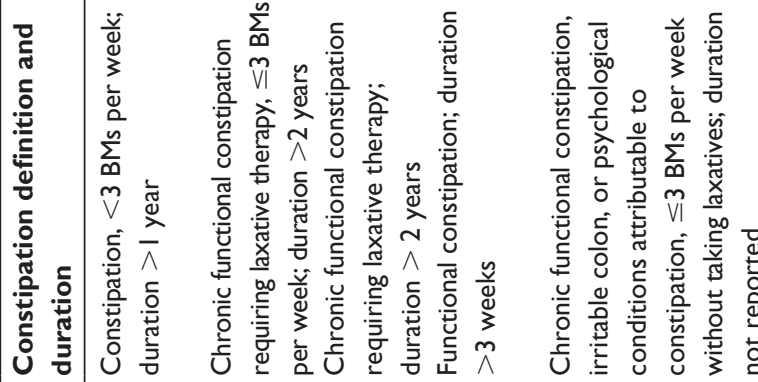

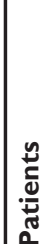

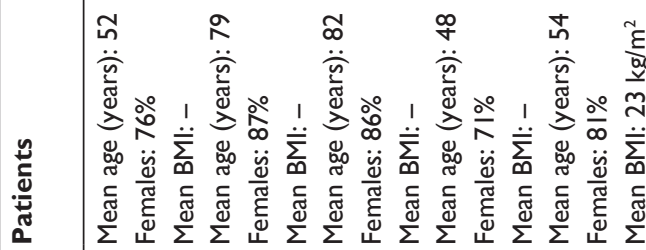

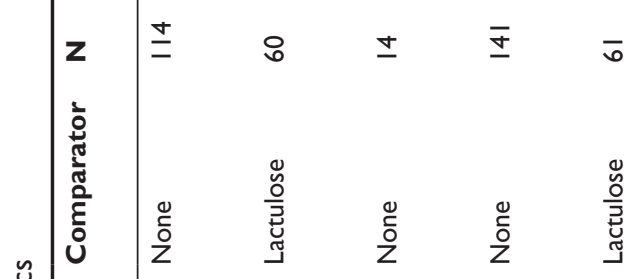

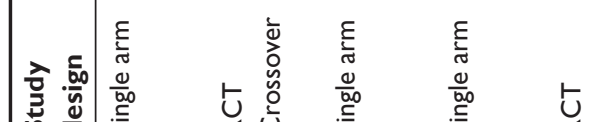

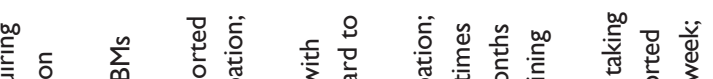
言紫

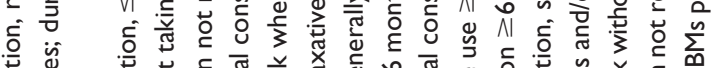

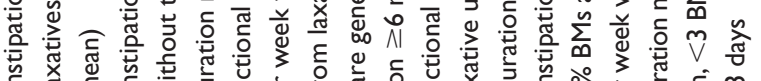

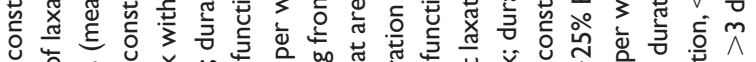

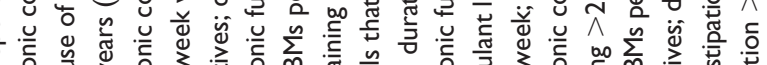

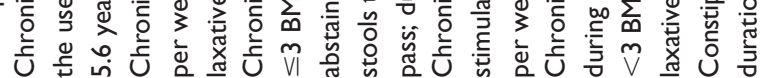

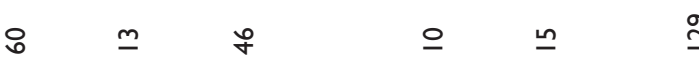

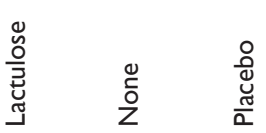

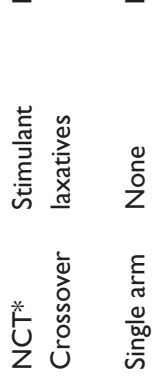

ฐิ

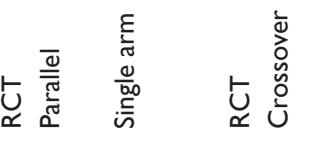

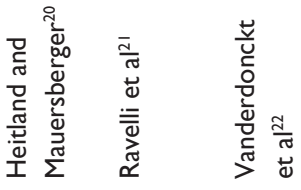

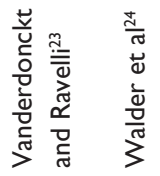

$\underset{\frac{\pi}{\pi}}{\frac{\pi}{\tilde{x}}}$

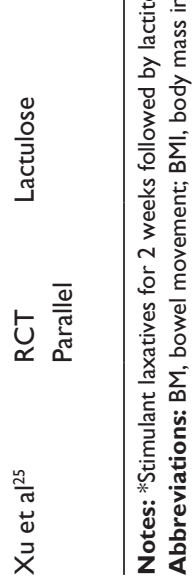




\begin{tabular}{|c|c|c|c|c|}
\hline \multirow[t]{2}{*}{ Study name } & \multicolumn{4}{|c|}{ Statistics for each study } \\
\hline & SMD & Lower limit & Upper limit & $P$-value \\
\hline Doffoel et al, ${ }^{17}$ & 2.035 & 1.584 & 2.486 & 0.000 \\
\hline Goovaerts et al, ${ }^{18}$ & 2.334 & 1.972 & 2.696 & 0.000 \\
\hline Hammer et al, ${ }^{19}$ & 1.344 & 0.859 & 1.830 & 0.000 \\
\hline Heitland et al, ${ }^{20}$ & 1.172 & 0.707 & 1.637 & 0.000 \\
\hline Ravelli et al, ${ }^{21}$ & 0.524 & -0.107 & 1.154 & 0.103 \\
\hline Vanderdonckt et al, ${ }^{22}$ & 0.545 & 0.225 & 0.865 & 0.001 \\
\hline Vanderdonckt et al, ${ }^{23}$ & 1.045 & 0.183 & 1.906 & 0.017 \\
\hline Walder et al, ${ }^{24}$ & 2.864 & 1.721 & 4.007 & 0.000 \\
\hline Xu et al, ${ }^{25}$ & 2.385 & 1.900 & 2.869 & 0.000 \\
\hline Overall & 1.555 & 0.998 & 2.112 & 0.000 \\
\hline
\end{tabular}

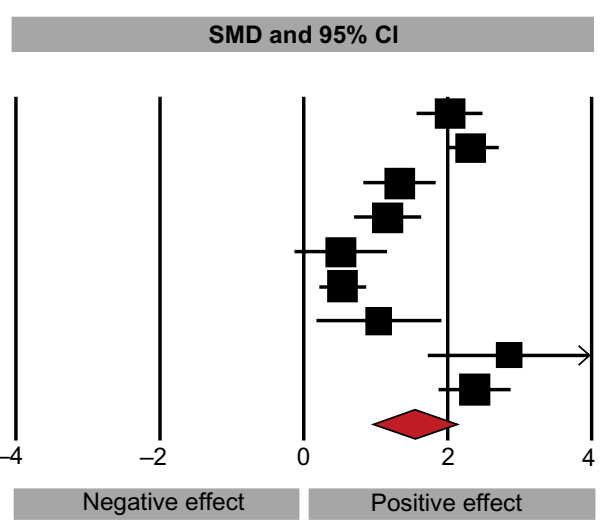

Figure 2 Standardized mean difference (SMD) for change in stool frequency with lactitol supplementation.

Notes: Data extracted from the lactitol arm of each included study, regardless of study design. $l^{2}=91 \%$, Egger's $P=0.67$.

Abbreviation: $\mathrm{Cl}$, confidence interval.

We performed univariate meta-regression to identify predictors of improvement in weekly stools with lactitol. Sample size, mean age, percentage of female patients, lactitol mean dose, and treatment duration were loaded into the model. Shorter treatment duration $\left(R^{2}=48 \%\right.$, $P<0.001)$, larger sample size $\left(R^{2}=41 \%, P<0.001\right)$, lower mean dose $\left(R^{2}=32 \%, P<0.001\right)$, younger age $\left(R^{2}=30 \%\right.$, $P<0.001)$, and higher percentage of female patients $\left(R^{2}=5 \%, P=0.03\right)$ were associated with more frequent defecation with lactitol supplementation. When dichotomizing predictor variables above and below median values for subgroup analyses, all treatment effects remained very large, with no statistically significant differences identified between subgroups. Specifically, stool frequency SMDs were 2.05 for treatment duration $<20$ days versus 1.17 for $\geq 20$ days $(P=0.12), 1.98$ for age $<50$ years versus 1.23 for age $\geq 50$ years $(P=0.14), 1.82$ for sample sizes $\geq 40$ versus 1.32 for $<40(P=0.41), 1.63$ for studies $\geq 75 \%$ female versus 1.51 for studies $<75 \%$ female ( $P=0.84$ ), and 1.29 for mean dose $\geq 20 \mathrm{~g}$ per day versus 1.34 for mean dose $<20 \mathrm{~g}$ per day $(P=0.95)$. Overall, lactitol similarly improved stool frequency across subgroups with possible additional benefit in younger adults and with shorter treatment durations.

\section{RCTs: lactitol versus lactulose}

Lactitol was slightly more effective than lactulose in increasing weekly stool frequency (SMD: $0.19,95 \% \mathrm{CI}:-0.01$ to $0.39, P=0.06$; Figure 4), with a difference in means of 0.3 (95\% CI: -0.1 to $0.8, P=0.15$ ) stools per week. Stool consistency was similar with lactitol and lactulose (SMD: 0.03, 95\% CI: -0.22 to $0.28, P=0.81$ ) (Figure 5). Only one study reported comparative product satisfaction assessments. ${ }^{19}$ Physician-assessed efficacy was $60 \%$ with lactitol and $64 \%$ with lactulose; patient-assessed efficacy was $60 \%$ for lactitol and $56 \%$ for lactulose. No statistically significant differences were noted for any tolerance outcome comparing lactitol with lactulose, with odds ratios of 0.81 (95\% CI: 0.37-1.76, $P=0.59)$ for AEs, 1.19 (95\% CI: 0.31-4.61, $P=0.80$ ) for AErelated withdrawals, and 0.94 ( $95 \% \mathrm{CI}: 0.26-3.33, P=0.92)$ for withdrawal for any reason. No SAEs were reported in any RCT comparing lactitol with lactulose. No significant heterogeneity or publication bias was identified for any outcome.

Study name
Goovaerts et al, ${ }^{18}$
Hammer et al, ${ }^{19}$
Ravelli et al, ${ }^{21}$
Vanderdonckt et al, ${ }^{22}$
Vanderdonckt et al, ${ }^{23}$
Xu et al, ${ }^{25}$
Overall

$\begin{array}{ll} & \text { Statistics } \\ \text { SMD } & \text { Lower limit } \\ 0.327 & 0.134 \\ 1.867 & 1.273 \\ 1.278 & 0.482 \\ 0.540 & 0.220 \\ 0.479 & -0.252 \\ 2.899 & 1.497 \\ 1.044 & 0.494\end{array}$

\section{for each study}

Upper limit $P$-value

0.520

2.461

2.075

0.859

1.211

4.301

1.594

0.001
0.000
0.002
0.001
0.199
0.000
0.000

$-4$

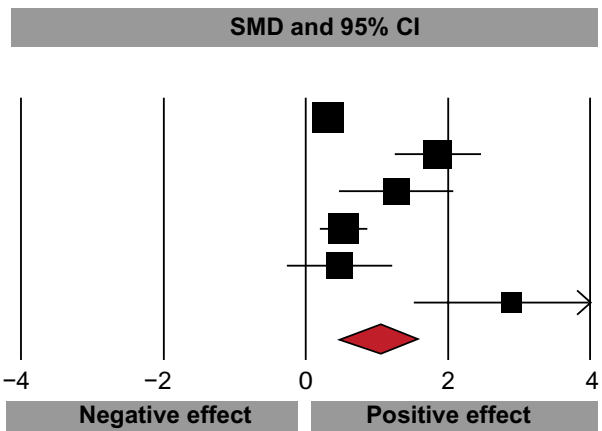

Figure 3 Standardized mean difference (SMD) for change in stool consistency with lactitol supplementation.

Notes: Data extracted from the lactitol arm of each included study, regardless of study design. $P^{2}=87 \%$, Egger's $P<0.05$.

Abbreviation: $\mathrm{Cl}$, confidence interval. 


\begin{tabular}{|c|c|c|c|c|}
\hline \multirow[t]{2}{*}{ Study name } & \multicolumn{4}{|c|}{ Statistics for each study } \\
\hline & SMD & Lower limit & Upper limit & $P$-value \\
\hline Doffoel et al, ${ }^{17}$ & 0.259 & -0.002 & 0.521 & 0.052 \\
\hline Hammer et al, ${ }^{19}$ & -0.165 & -0.687 & 0.357 & 0.536 \\
\hline Heitland et al, ${ }^{20}$ & 0.468 & -0.045 & 0.981 & 0.074 \\
\hline $\mathrm{Xu}$ et $\mathrm{al},{ }^{25}$ & 0.109 & 0.237 & 0.454 & 0.537 \\
\hline Overall & 0.189 & -0.009 & 0.387 & 0.061 \\
\hline
\end{tabular}

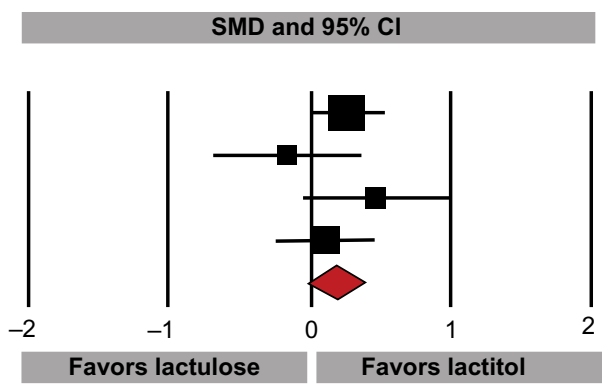

Figure 4 Standardized mean difference (SMD) for change in stool frequency comparing lactitol with lactulose.

Notes: Data extracted from four randomized controlled trials comparing lactitol to lactulose. $I^{2}=11 \%$, Egger's $P=0.74$.

Abbreviation: $\mathrm{Cl}$, confidence interval.

\section{RCT: lactitol versus placebo}

A single randomized, placebo-controlled clinical trial of lactitol in constipated adults has been performed. ${ }^{22}$ Forty-six elderly patients (mean age: 84 years) were randomly assigned to 4 weeks of lactitol or placebo in a crossover design. At baseline, mean weekly stool frequency was $2.4 \pm 0.6$ and 11 (26\%) of 43 patients reported hard stools. Over the 4-week intervention period, stool frequency improved by approximately 2.5 stools per week with lactitol relative to placebo $(P<0.001)$. Stool consistency improved by approximately 0.5 points (on a four-point scale) with lactitol relative to placebo $(P=0.001)$. The incidence of side effects, including flatulence, bloating, abdominal cramps, or diarrhea, was 51\% (22/43) with lactitol and $30 \%(13 / 43)$ with placebo $(P=0.08)$. No AEs were classified as serious and none led to patient withdrawal from the study.

\section{Nonrandomized controlled trial: lactitol versus stimulant laxative}

Vanderdonckt and Ravelli ${ }^{23}$ conducted a nonrandomized crossover study in which ten elderly patients (mean age: 75 years) took stimulant laxatives for 2 weeks followed by lactitol for 4 weeks. Lactitol resulted in a higher weekly stool frequency compared with stimulant laxatives $(2.0 \pm 2.8, P=0.03)$.
Physician-rated efficacy of lactitol was favored over stimulant laxatives in $8 / 10$ patients and lactitol tolerance was favored in $7 / 10$ patients. All ten patients completed the 2-week stimulant laxative phase, but two patients discontinued lactitol due to abdominal discomfort.

\section{Discussion}

We performed the first meta-analysis on the efficacy and tolerance of lactitol in the treatment of adult constipation. Overall, lactitol supplementation was found to be well tolerated and to improve stool frequency and consistency in constipated patients. While gastrointestinal side effects were common, they were generally mild in nature. The efficacy and tolerance of lactitol for adult constipation were found to be similar to those of lactulose.

Although excluded from the current meta-analysis, two relevant studies also suggested favorable laxative effects with lactitol for adult constipation attributed to surgery or hospitalization. Copé et $\mathrm{al}^{26}$ conducted an RCT of lactitol or oral petroleum jelly for recovery of intestinal transit following anal surgery. Patients consuming $20 \mathrm{~g}$ lactitol per day had a quicker time to first stool after surgery (24 versus 39 hours, $P=0.002$ ) versus those assigned to petroleum

\begin{tabular}{lllll}
\multicolumn{1}{c}{ Study name } & \multicolumn{5}{c}{ Statistics for each study } \\
\cline { 2 - 5 } & SMD & Lower limit & Upper limit & $P$-value \\
Doffoel et al, ${ }^{17}$ & 0.069 & -0.315 & 0.453 & 0.723 \\
Hammer et al, $^{19}$ & 0.253 & -0.436 & 0.943 & 0.471 \\
Heitland et al, $^{20}$ & -0.207 & -0.872 & 0.458 & 0.543 \\
Xu et al, ${ }^{25}$ & -0.008 & -0.453 & 0.436 & 0.970 \\
Overall & 0.031 & -0.218 & 0.279 & 0.810
\end{tabular}

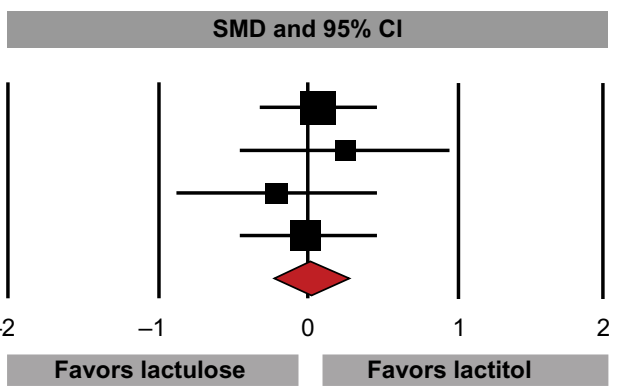

Figure 5 Standardized mean difference (SMD) for change in stool consistency comparing lactitol with lactulose.

Notes: Data extracted from four randomized controlled trials comparing lactitol to lactulose. $I^{2}=0 \%$, Egger's $P=0.92$.

Abbreviation: $\mathrm{Cl}$, confidence interval. 
jelly and reported more frequent bowel movements over the 7 -day postoperative period. Pontes et $\mathrm{al}^{27}$ conducted an RCT comparing liquid lactulose, crystallized lactulose, crystallized lactitol, and a control in hospitalized patients without reported constipation. All active therapies decreased colonic transit time compared with controls, with the lactulose groups experiencing a slightly greater improvement compared with lactitol.

The conclusions of the current meta-analysis differ somewhat compared with those of two earlier systematic reviews, ${ }^{28,29}$ mainly due to their inclusion of non-peerreviewed reports, studies with children, or adults with hospitalization- or surgery-related constipation. Both of those systematic reviews reported superior efficacy and better tolerance of lactitol over lactulose. Based on a systematic review of peer-reviewed manuscripts in adults with functional constipation, we observed a trend for improved stool frequency with lactitol versus lactulose but no differences in other efficacy or tolerance parameters. Several authors have reported that the excessive sweetness of lactulose negatively affects patient compliance and that the slightly sweet taste of lactitol is preferred by patients. ${ }^{30-32}$ While several single-arm studies in the current meta-analysis reported excellent patient acceptance with lactitol, no RCTs with lactitol or lactulose reported data on taste preference and no statistically significant differences were identified in any tolerance outcome.

Although lactitol is generally well tolerated up to $40 \mathrm{~g}$ per day, ${ }^{33}$ mild gastrointestinal complaints are common, with a similar frequency to lactulose and a nonsignificantly higher frequency compared with placebo. Only $5 \%$ of patients discontinued lactitol therapy due to gastrointestinal side effects, with no differences noted between lactitol and lactulose or placebo. The laxative effect with lactitol is attributed to a lowering of colonic $\mathrm{pH}$ as a consequence of fermentation by the colonic microbiota, stimulation of peristalsis, and increased osmotic pressure in the intestinal lumen, ultimately resulting in an increase in fecal volume and more frequent defecations. ${ }^{11}$ Although this mechanism of action may result in mild gastrointestinal complaints in some patients, the benefit-to-risk profile of lactitol remains favorable given the significant laxative effect in constipated patients.

There were several limitations to this meta-analysis. First, despite the strong efficacy profile of lactitol, the limited number of controlled studies made interpretation of the comparative benefits difficult. Second, 10/11 studies were published between 1988 and 1995. Although numerous gastrointestinal studies have been recently conducted with lactitol, it is unclear why there is a dearth of recent studies in adult constipation. Third, the number of included studies was minimally adequate to execute meta-regressions and subgroup analyses; therefore, these estimates may be somewhat unstable. Fourth, duration of constipation symptoms varied among studies and was occasionally unreported. Finally, only two studies administered lactitol for over 4 weeks. Therefore, the effects of long-term lactitol use for treatment of adult constipation remain unknown.

\section{Conclusion}

Lactitol supplementation was found to be well tolerated and to significantly improve symptoms of adult constipation. The efficacy and tolerance of lactitol and lactulose were comparable, with a trend for more frequent stools with lactitol. Limited evidence suggests lactitol is superior to stimulant laxatives and placebo for relieving constipation symptoms. The small number of comparative studies with lactitol is a major limitation. We recommend additional larger scale RCTs to further characterize the effect of lactitol supplementation in this patient population, with recommended comparisons including placebo controls, other osmotic laxatives, fibers, and probiotics.

\section{Disclosure}

This research was supported by Danisco Sweeteners Oy (Kantvik, Finland). JT and ACO are employees of Danisco Sweeteners; Danisco Sweeteners manufactures lactitol. LEM declares no conflict of interest.

\section{References}

1. Stewart WF, Liberman JN, Sandler RS, et al. Epidemiology of constipation (EPOC) study in the United States: relation of clinical subtypes to sociodemographic features. Am J Gastroenterol. 1999;94(12): 3530-3540.

2. Sonnenberg A, Koch TR. Epidemiology of constipation in the United States. Dis Colon Rectum. 1989;32(1):1-8.

3. Sanchez MI, Bercik P. Epidemiology and burden of chronic constipation. Can J Gastroenterol. 2011;25 Suppl B:11B-15B.

4. Rome Foundation. Guidelines - Rome III Diagnostic Criteria for Functional Gastrointestinal Disorders. J Gastrointestin Liver Dis. 2006;15(3):307-312

5. Dennison C, Prasad M, Lloyd A, Bhattacharyya SK, Dhawan R, Coyne K. The health-related quality of life and economic burden of constipation. Pharmacoeconomics. 2005;23(5):461-476.

6. Nyrop KA, Palsson OS, Levy RL, et al. Costs of health care for irritable bowel syndrome, chronic constipation, functional diarrhoea and functional abdominal pain. Aliment Pharmacol Ther. 2007;26(2):237-248.

7. Andrews CN, Storr M. The pathophysiology of chronic constipation. Can J Gastroenterol. 2011;25 Supp1 B:16B-21B.

8. Johanson JF, Kralstein J. Chronic constipation: a survey of the patient perspective. Aliment Pharmacol Ther. 2007;25(5):599-608.

9. Tack J, Muller-Lissner S, Stanghellini V, et al. Diagnosis and treatment of chronic constipation - a European perspective. Neurogastroenterol Motil. 2011;23(8):697-710. 
10. Luciano KL. Diagnosis and management of functional constipation in children. JAAPA. 2013;26(12):21-24.

11. Schiller LR. Review article: the therapy of constipation. Aliment Pharmacol Ther. 2001;15(6):749-763.

12. Cohen J. Statistical Power Analysis for the Behavioral Sciences. Hillside, NJ: Erlbaum; 1987.

13. Higgins JP, Thompson SG, Deeks JJ, Altman DG. Measuring inconsistency in meta-analyses. BMJ. 2003;327(7414):557-560.

14. Egger M, Davey Smith G, Schneider M, Minder C. Bias in meta-analysis detected by a simple, graphical test. BMJ. 1997;315(7109):629-634.

15. Delas N, Gislon J, Glikmanas M, et al. [Lactitol in the treatment of constipation in the adult. Open, non-comparative study of its efficacy and its clinical and biological tolerance]. Ann Gastroenterol Hepatol (Paris). 1991;27(5):231-233. French.

16. Doffoel M, Berthel M, Bockel R, Kuntzman F, Brunet CM. [Clinical investigation of the long-term tolerability ( 6 months) of lactitol for the treatment of functional constipation in elderly patients]. Médecine et Chirurgie Digestives (Paris). 1990;19(4):260-261.

17. Doffoel M, Berthel M, Bockel R, Kuntzman F, Brunet CM. [Comparative study of lactitol and lactulose in the treatment of functional constipation in elderly subjects]. Médecine et Chirurgie Digestives (Paris). 1990;19(4):257-259. French.

18. Goovaerts L, Ravelli GP. Lactitol monohydrate for the treatment of chronic constipation: a multicentre study on the efficacy and tolerability of an individually adjusted daily dose. Acta Therapeutica. 1993;19:61-69.

19. Hammer B, Ravelli GP. [Chronic functional constipation: lactitol maintenance dose, a multicentre comparative study with lactulose]. Ther Schweiz. 1992;8:328-335. German.

20. Heitland $\mathrm{W}$, Mauersberger $\mathrm{H}$. [A clinical investigation comparing the laxative effect of lactitol to that of lactulose in a randomised, open, parallel study]. Schweiz Rundsch Med Prax. 1988;77(18):493-495. German.

21. Ravelli GP, Whyte A, Spencer R, Hotten P, Harbron C, Keenan R. The effect of lactitol intake upon stool parameters and the faecal bacteria flora in chronically constipated women. Acta Therapeutica. 1995;21: $243-254$.
22. Vanderdonckt J, Coulon J, Denys W, Ravelli GP. Study of the laxative effect of lactitol (Importal) in an elderly institutionalized, but not bedridden, population suffering from chronic constipation. Journal of Clinical and Experimental Gerontology. 1990;12(3):171-189.

23. Vanderdonckt J, Ravelli GP. Lactitol as an alternative to harsh (irritant) laxatives: an exploratory, open pilot study in chronic functional constipation. Acta Therapeutica. 1993;19:295-308.

24. Walder M, Buclin T, Biollaz J, Kitler ME, Schelling JL. [Dose-response curve and preliminary clinical study of a laxative, lactitol]. Schweiz Med Wochenschr. 1988;118:1925-1927. French.

25. Xu Z, Dai J, Shi R, et al. A multicenter, randomized, single-blinded, parallel-controlled trial on lactitol in treatment of constipation. Chinese Journal of Gastroenterology. 2012;17(3):168-172.

26. Copé R, Debou JM, Cohen S, Callens A. [Study of early postoperative lactitol versus paraffin following anal surgery in 110 adult patients]. Ann Chir. 1996;50(7):570-576.

27. Pontes FA, Silva AT, Cruz AC. Colonic transit times and the effect of lactulose or lactitol in hospitalized patients. Eur J Gastroenterol Hepatol. 1995;7(5):441-446.

28. Maydeo A. Lactitol or lactulose in the treatment of chronic constipation: result of a systematic. J Indian Med Assoc. 2010;108(11):789-792.

29. Faruqui AA, Joshi C. Lactitol: a review of its use in the treatment of constipation. Int J Recent Adv Pharm Res. 2012;2(1):1-5.

30. Pai CH, Huang YS, Jeng WC, Chan CY, Lee SD. Treatment of porto-systemic encephalopathy with lactitol verus lactulose: a randomized controlled study. Zhonghua Yi Xue Za Zhi (Taipei). 1995;55(1):31-36.

31. Heredia D, Terés J, Orteu N, Rodés J. Lactitol lactulose in the treatment of chronic recurrent portal-systemic encephalopathy. J Hepatol. 1988;7(1):106-110.

32. Lanthier PL, Morgan MY. Lactitol in the treatment of chronic hepatic encephalopathy: an open comparison with lactulose. Gut. 1985;26(4):415-420.

33. Patil DH, Grimble GK, Silk DB. Lactitol, a new hydrogenated lactose derivative: intestinal absorption and laxative threshold in normal human subjects. Br J Nutr. 1987;57(2):195-199.
Clinical and Experimental Gastroenterology

\section{Publish your work in this journal}

Clinical and Experimental Gastroenterology is an international, peerreviewed, open access journal, publishing all aspects of gastroenterology in the clinic and laboratory, including: Pathology, pathophysiology of gastrointestinal disease; Investigation and treatment of gastointestinal disease; Pharmacology of drugs used in the alimentary tract;

\section{Dovepress}

Immunology/genetics/genomics related to gastrointestinal disease. This journal is indexed on CAS. The manuscript management system is completely online and includes a very quick and fair peer-review system. Visit http://www.dovepress.com/testimonials.php to read real quotes from published authors. 